This item was submitted to Loughborough's Research Repository by the author.

Items in Figshare are protected by copyright, with all rights reserved, unless otherwise indicated.

\title{
A distribution-sensitive poverty index-based protection scheme for interconnected DC microgrids
}

PLEASE CITE THE PUBLISHED VERSION

https://doi.org/10.1049/cp.2020.0111

PUBLISHER

IET

VERSION

AM (Accepted Manuscript)

\section{PUBLISHER STATEMENT}

This paper is a postprint of a paper submitted to and accepted for publication in 15th International Conference on Developments in Power System Protection (DPSP 2020) and is subject to Institution of Engineering and Technology Copyright. The copy of record is available at the IET Digital Library.

\section{LICENCE}

CC BY-NC-ND 4.0

\section{REPOSITORY RECORD}

Bayati, N, A Hajizadeh, M Soltani, and Zhengyu Lin. 2021. "A Distribution-sensitive Poverty Index-based Protection Scheme for Interconnected DC Microgrids". Loughborough University. https://hdl.handle.net/2134/14837820.v1. 


\title{
A Distribution-Sensitive Poverty Index-Based Protection Scheme for Interconnected DC Microgrids
}

\author{
Navid Bayati ${ }^{*}$, Amin Hajizadeh ${ }^{1}$, Mohsen Soltani ${ }^{1}$,Zhengyu Lin ${ }^{2}$ \\ ${ }^{1}$ Department of Energy Technology, Aalborg University, Esbjerg, Denmark \\ ${ }^{2}$ School of Mechanical, Electrical and Manufacturing Engineering, Loughborough University, Loughborough, \\ U.K. \\ *nab@et.aau.dk
}

Keywords: DC Microgrid, Protection, Fault, Interconnected Microgrids, Distribution-Sensitive Poverty Index

\begin{abstract} in different scenarios.

\section{Introduction}

Due to the increasing the integration of DC renewable energy sources (RESs) such as fuel cells and photovoltaic (PVs) arrays, and modern DC loads, DC Microgrids can be widely utilized to increase efficiency and reliability of future grids [1]. The advantages of DC Microgrids are the elimination of harmonics and reactive power issues, not requiring the frequency and synchronization control, reduced power conversion stages [2]. The DC Microgrid can be operated as two different operational modes, islanded mode, and grid-connected mode [3] which is connected to the grid by converters. Moreover, increasing the integration and attention to DC Microgrids causes the interconnecting of DC Microgrids to make a high power interconnected DC Microgrid network. Due to the presence of different DC Microgrids in these systems, the complexity of protection, control, and monitoring of these systems is a challenge.
\end{abstract}

In recent years, due to the increasing penetration of renewable energy resources, the concept of DC Microgrid is determined. In addition, the lack of effective protection strategy and standards for DC Microgrids jeopardizes the reliability of these systems. In this paper, a protection scheme for interconnected DC Microgrids by using a distribution sensitive poverty index is proposed. This strategy is designed by monitoring of current of interconnected line and sending to a central protection unit to detect and isolate the faulty DC Microgrids. The proposed method is able to detect and isolate DC Microgrids in case of the line to ground and line to line faults in case of low and high impedance faults. The effectiveness of the proposed method is proved by the implementation of an interconnected DC Microgrid in MATLAB. The results show the speed and capability of fault detection

On the other hand, due to the nature of DC current during the fault, lack of standards, and effective protection strategy, protection of DC Microgrids during fault is one of the operational challenges [4]. There are two types of faults in the DC systems, line to line faults, which causes when two lines are connected to each other, and line to ground [5] that causes during connecting of a line to ground. Fault in DC system has high amplitude and fast rise rate that require fast fault detection [6-7]. Therefore, designing a fast, reliable, and low-cost protection strategy is essential for reduction of the operational risk in DC Microgrids [8].

The suggested protection scheme in [9] is based on the transient behavior of first and second-order derivatives of the DC current during the fault. In [10], an overcurrent relay is used as a hybrid passive protection device by using the current, and voltage transducers and real-time discrete wavelet transform to detect the fault. In [11], a non-iterative fault location method is proposed by using the probe power unit. In [12], a protection method is proposed to detect the zone of fault in a DC Microgrid by using the minimum number of protection devices and measurement units. Changing direction and amplitude of current during fault is the basis of the protection method of [13]. In [14], it is suggested to inject a short duration current to the DC bus of the maritime DC Microgrids to measure the bus impedance and detect the fault, but, it is an active and costly fault protection method. The non-unit protection methods can detect only the low impedance faults and require additional equipment. Therefore, the majority of faults, which have high impedance faults (HIFs), cannot be detected by these methods.

Moreover, other proposed methods are focused on enhancing the speed and sensitivity of the protection systems of DC Microgrids by using communication channels. In [15], the differential relay uses communication links to monitor the magnitude of the current and detect the fault. Ref. [16] uses the least square method to estimate the fault parameters and determine the fault direction using the information of both ends of the line. Also, in [17-18], the differential protection scheme is proposed based on the modified cumulative sum average method for DC Microgrid protection. A protection strategy based on the direction of the current by using the data of both ends of the protection zone is presented in [19].

Detecting the HIFs is one of the main challenges of the protection system because the fault current changes of the HIFs are not significantly high and it is close to overload [20]. During the HIFs, the fault current direction can be unchanged, and therefore, the fault imposed current would be lower than the 
pre-fault current [21]. To design an active protection system for solving this problem, [22] employs the transient fault current components. However, this method cannot protect a DC Microgrid with different zones during the HIF.

On the other hand, in the systems with different DC Microgrids, the complexity of the system and also the bidirectional current between DC Microgrids increases the difficulty of proposing a protection system for isolating a DC Microgrid during the fault. Also, the lack of enough study in this issue, and low tolerant of DC/DC converters between DC Microgrids increase the importance of solving this problem. To address the aforementioned problems, in this paper a directional protection scheme for interconnected DC Microgrids is proposed, which uses the current samples between the DC Microgrids to isolate the faulty zone. Each interconnection line between DC Microgrids has two relays at both ends of the line to calculate the distribution-sensitive poverty index (DSPI) to measure the fault condition severity using the transient performance of the fault current. It is notable that it is the first time that DSPI is applied for the protection of an interconnected DC Microgrid.

Based on the DSPI value of each relay, the faulty zone is detected and isolated within milliseconds and is also considered the HIFs. The proposed protection scheme includes a localized relay for determining the DSPI, a circuit breaker, and a measurement unit at each end of the interconnected lines. The main features of the paper are as follows:

- Proposing a fault detection method with high sensitivity on the HIFs based on the DSPI.

- $\quad$ Localizing the protection system

- $\quad$ Protecting an interconnected DC Microgrid system consists of several individual DC Microgrids.

- $\quad$ Proposing a protection method for both islanded and grid-connected modes.

The rest of this paper is organized as follows: Section II presents the characteristics and challenges of an interconnected DC Microgrid. In section III, the proposed scheme is presented. The simulation results and discussions are presented in section IV. Finally, the conclusions are given in section V.

\section{Interconnected DC Microgrids}

The bidirectional current in interconnected DC Microgrids causes several challenges during the fault conditions. An interconnected microgrid architecture is presented in [23], with a power management system. In [24], the power management of these systems is proposed and evaluated. However, in the aforementioned literature, the impact of the fault and also the protection system for the interconnected DC Microgrids are not considered. DC Microgrids can be connected to each other and make an interconnected DC Microgrid network. These systems improve reliability and stability. On the other hand, interconnecting DC Microgrids to each other causes some protection problems, such as increasing the short circuit level, the complexity of coordination of protection devices of each DC Microgrid, and the impact of the internal fault of a DC Microgrid to the entire of the cluster. There is a lack of enough study on the protection of these systems. The general structure of an interconnected DC Microgrid network is shown in Fig. 1.

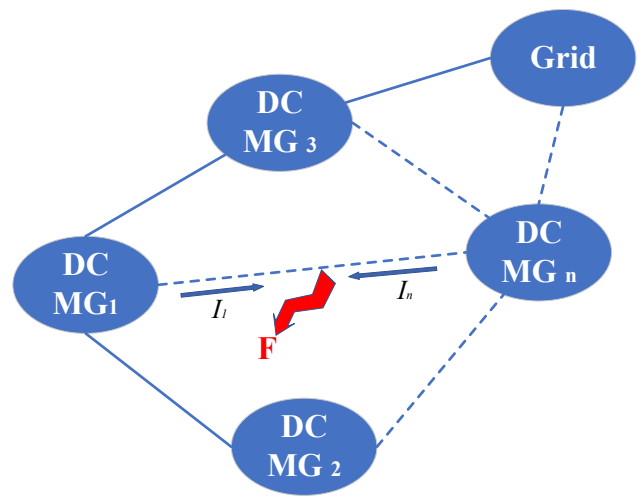

Fig. 1 The general diagram of an interconnected DC Microgrid

As shown in Fig. 1, several DC Microgrids are connected to each other by a line, and the finite number of DC Microgrids are connected to the grid by a DC/AC converter. During a fault at the line between DC Microgrids 1 and $n$, the fault current is injected from both ends of the faulty line and can change the current flows of other interconnected lines. Changing the fault flows of other interconnected lines increases the complexity of designing a protection strategy. Also, due to the high number of points of connections and RESs in this system, the short circuit level of an interconnected DC Microgrid network is significantly higher than a standalone DC Microgrid. Moreover, this high number of components causes more variations in the output power of RESs, which causes more variations in the value of fault current. In summery the different components of an interconnected DC Microgrid are introduced in this section.

\subsection{RESS}

The main RESs in the DC Microgrids are PVs and wind turbines (WTs). PV is mainly modeled by the equivalent circuit, which divided into four different models, namely, ideal, single diode with series resistant, single diode with parallel resistant, and two diodes model. For the protection studies, most accurate model is two-diode model [25], which gives an accurate current during the fault. During the fault, PV starts to inject a current into the fault location, and the value of this current depends on the structure of the converter. If the PV equipped with a maximum power point tracker, the behavior of the PV and its converter will be the same as a constant power source. Also, the limitation strategy of converter limits the value of fault current to twice the maximum current rating [26]. Another most utilized RESs is WT, which a WT should be connected to an AC/DC converter for connecting to the DC Microgrid. The protection strategy of a WT converter limits the current of WT during the fault. 


\subsection{Converter}

The behavior of a power converter during the fault in a DC Microgrid is divided into a passive discharge current by capacitors and an uncontrollable fault current by RESs. The modular multilevel converter (MMC) is widely used in the DC Microgrids. The fault current is affected by two aspects of the MMCs. In the first one, the distributed capacitors on the MMC cause a capacitor discharge current during the fault, and the second one, the series reactors of the MMC limit the rising rate of the fault current. The converter simplified model that considers the capacitor discharge effect of the MMCs is appropriate for fault studies, and also should consider the decreasing trend of the converter voltage.

\subsection{Energy Storage}

Due to the uncertainty of RESs in DC Microgrids, energy storages are necessary for these systems. The simplified equivalent circuit of the battery energy storage system consists of a resistor paralleled with a capacitor in series with a voltage source to provide an accurate dynamic of this component during the fault. The paralleled RC represents the nonlinear polarization of the battery. The value of the voltage source is the open-circuit voltage of the battery. Due to the presence of capacitor in this model, during the first moments of fault, capacitor discharges a fault current into the system, and the voltage source supplies a constant value of fault current.

\section{Proposed Protection Strategy}

The bidirectional power flow in an interconnected DC Microgrid cause the challenges for the protection methods. In addition, due to the no significant changes in the direction and magnitude of the fault current during HIFs degrade the appropriately of a directional protection strategy. To solve these problems, a directional-based protection scheme is proposed in this paper which uses the transient measured values of current at the interconnected lines between the DC Microgrids. In the first step, the variation value of the current during fault is determined. During normal condition, this value is zero. Due to the low changes of HIFs, in this paper the DSPI in used to overcome this problem. By calculating the DSPI variation during the fault, the proposed strategy can accurately detect the faulty DC Microgrid. The details of the proposed protection scheme are explained in this section.

\section{A. Distribution-Sensitive Poverty Index}

The distribution-sensitive poverty index (DSPI) was proposed to measure poverty in a certain population [27]. This algorithm is sensitive to the income distribution. This paper applies the concept of the DSPI for measuring the change of current during the fault. At the first stage, the current of the interconnection line $I$ is measured by using a current measurement and filtered by a low pass filter to reduce the noise. The required sample rate for the current measuring is $5 \mathrm{kHz}$. By using the superposition theory [28], the fault-imposed components can be calculated by
$i_{F I}\left(k T_{s}\right)=i_{F}\left(k T_{s}\right)-i_{n}\left(k T_{s}\right)$

where, $i_{F I}$ is the fault imposed current, $i_{F}$ is the fault current, and $i_{n}$ is the current during the normal operation. By using the delta filter [29], the equation (1) can be modified to

$i_{F I}\left(k T_{s}\right)=i_{F}\left(k T_{s}\right)-i_{n}\left(k T_{s}-T_{d}\right)$

(2)

where, $T_{d}$ is the delay of filter.

The value of DSPI is calculated by

$D S P I=\frac{1}{N} \sum_{J=1}^{N}\left[\left(\ln (z)-\ln \left(i_{F I}\right)\right) \times\left(u\left(i_{F I}-z\right)\right)\right]$

where, $z$ is the poverty line, $u$ is the unit step function, and $N$ is the number of data. The value of $z$ is determined by the maximum value of the overload, which is typically $20 \%$ more than the normal load conditions. The value of DSPI for values less than the $z$ is zero. In the measurement, the value of noise due to the disturbances and loads variations may cause a maloperation, however, the presence of the poverty line reduces the impact of noises.

\section{B. Line Protection}

In this paper, a DSPI based directional protection strategy is proposed for protection of interconnection lines of DC Microgrid and isolating the faulty Microgrid. The proposed method should be installed in each line of the interconnected DC Microgrid, as shown in Fig. 2.

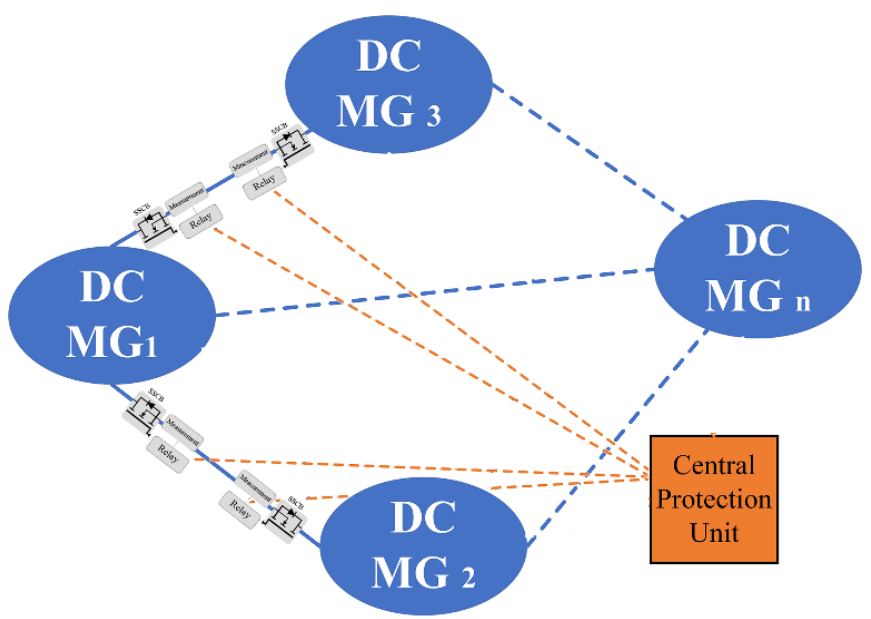

Fig. 2 The structure of the proposed protection strategy

The relays are connected to a central protection unit (CPU) to evaluate the states of each line and DC Microgrids. For interrupting the fault current, each line has two solid state circuit breakers (SSCBs). The relay of each side receives the measured current value and calculates the value of DSPI, and then send this value to the CPU for decision making. The proposed protection principle is determined by 


$$
\Delta D S P I=\left|D S P I_{1}-D S P I_{2}\right|
$$

where, $\mathrm{DSPI}_{1}$ and DSPI $\mathrm{D}_{2}$ are the DSPI's for the first and second ends of the line. When the value of DSPI differences exceeds the fault detection threshold $\varepsilon$, the fault is detected and the values of DSPIs send to the CPU for identification of the faulty DC Microgrid. On the other hand, after the fault detection, the direction of currents during the fault can be used to find the faulty DC Microgrid. The fault current directions for two different locations are shown in Fig. 3. During the fault, a variable for detecting the direction of fault current in each line are defined, $\lambda$. If the fault current is injected through the line $\mathrm{m}^{\text {th }}$ to $\mathrm{DC} \mathrm{MG}_{\mathrm{n}}$, the value of $\lambda_{\mathrm{nm}}$ will be 1 , and if the fault current injected through the line $\mathrm{m}^{\text {th }}$ from the $\mathrm{DC}_{\mathrm{MG}}$, the value of $\lambda_{\mathrm{nm}}$ will be -1 . Therefore, the values of $\lambda$ for DC Microgrids and lines send to the CPU. Consequently, if the value of $\triangle D S P I$ increases to more than the threshold, the fault is detected, and then the following equation calculated for each DC Microgrid

$$
K_{n}=\sum_{i=1}^{m} \lambda_{n i}
$$

where, $K_{n}$ identified the faulty DC Microgrid number $n$, if the value of $K_{n}$ become equal to the $-m$. The flowchart of the proposed strategy is shown in Fig. 4.

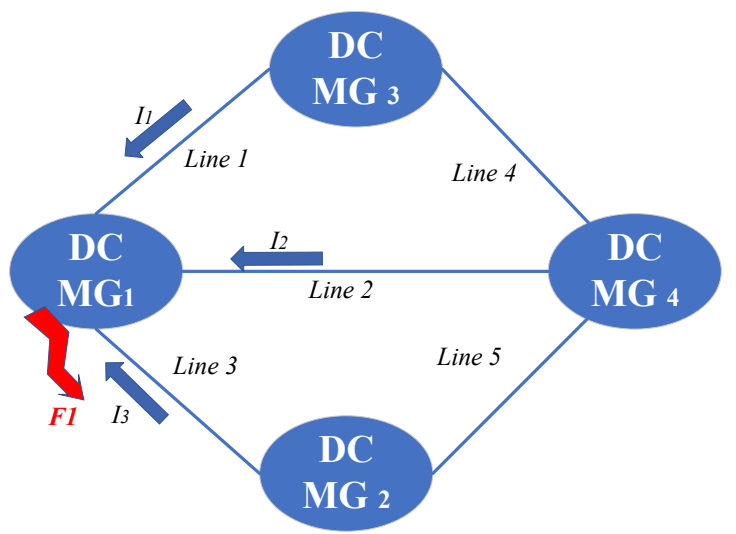

(a)

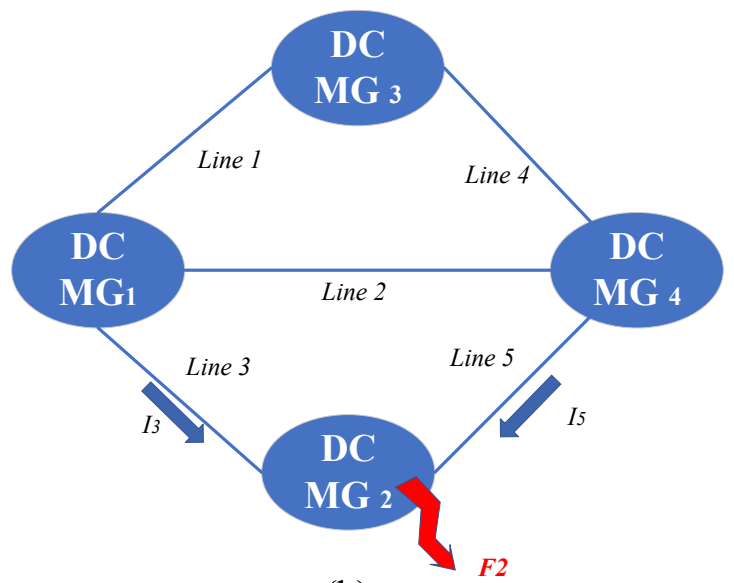

(b)

Fig. 3 Fault current through the lines (a) $\mathrm{F}_{1}$ : fault at the DC Microgrid 1 (b) $F_{2}$ : fault at the DC Microgrid 2

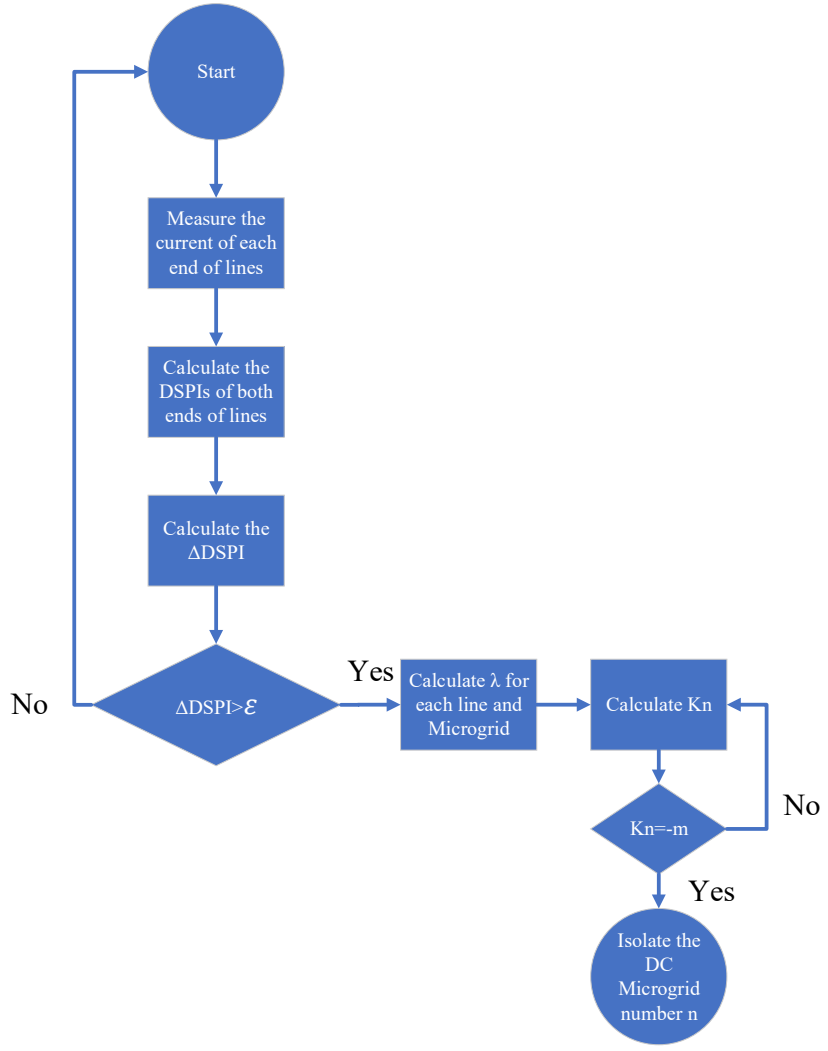

Fig. 4 Flowchart of the proposed method

\section{Simulation Results}

A set of simulation studies are performed to evaluate the performance of the proposed strategy under different scenarios. The case study DC network consists of three DC Microgrids, as shown in Fig 5, in which the total power generation of RESs and loads are represented in Table 1.

Table 1 Definition of case study

\begin{tabular}{lll}
\hline DC Microgrid & Type of RESs & Total Loads \\
\hline $\mathrm{DC} \mathrm{MG}_{1}$ & PV, WT, FC & $207 \mathrm{~kW}$ \\
$\mathrm{DC} \mathrm{MG}_{2}$ & WT, FC & $216 \mathrm{~kW}$ \\
$\mathrm{DC} \mathrm{MG}_{3}$ & WT, PV & $140 \mathrm{~kW}$ \\
\hline
\end{tabular}

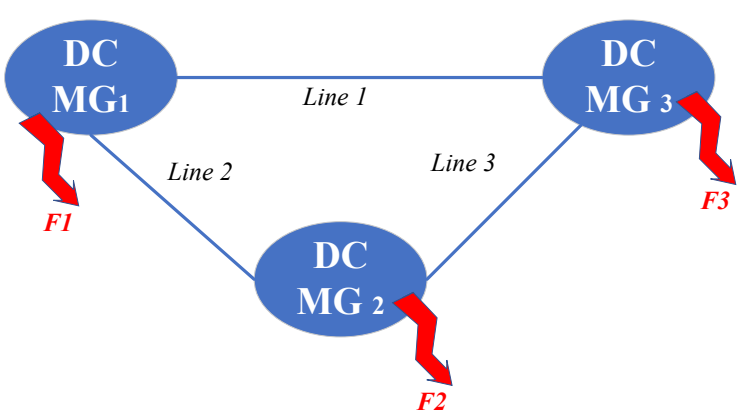

Fig. 5 Understudy interconnected DC Microgrid

Three different scenarios with different fault resistances are simulating on the understudy interconnected DC Microgrid. Each scenario presents the fault in each DC Microgrid. Based on the proposed method, the value of the $\triangle D S P I$ is calculated 
for each line. The value of threshold is defined based on the worst case of fault with highest fault resistance. In this paper, the value of thresholds for lines 1, 2, and 3 are 1.0705, 1.0770, and 1.0842 . The waveform of the fault current through the line 1 for $F_{1}$ by fault resistance of $0.3 \Omega$ is depicted in Fig 6, which the fault current rises to $817 \mathrm{~A}$. This high rise value of current can damage the converters and lines in the path. Therefore, by using the proposed method, the values of $\triangle D S P I$ for detecting the fault during the different scenarios are shown in Table 2. The results show that during all HIFs and different locations, the event of fault is detected by proposed method. In addition, the value of $z$ for calculating of DSPI is $20 \%$ more than the normal line current. The normal line currents of line $e_{1}$, line2, line 3 are 45.4, 79, 59 A, respectively.

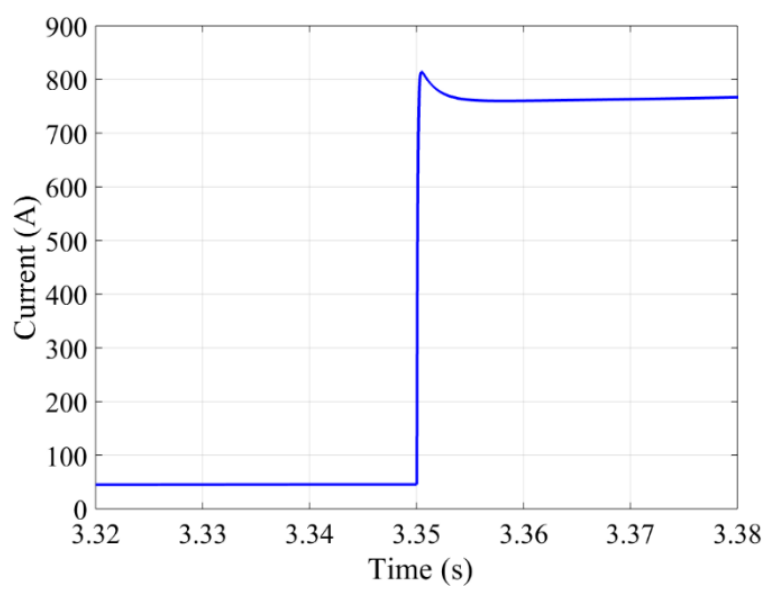

Fig. 6 Fault current of the interconnected line

Table 2 The values of $\triangle D S P I$ during different scenarios

\begin{tabular}{lllll}
\hline $\begin{array}{l}\text { Faulty } \\
\text { DC MG }\end{array}$ & $\begin{array}{l}\text { Fault } \\
\text { resistance }(\Omega)\end{array}$ & $\Delta$ DSPI $_{1}$ & $\Delta \mathrm{DSPI}_{2}$ & $\Delta \mathrm{DSPI}_{3}$ \\
\cline { 2 - 4 } $\mathrm{DC} \mathrm{MG}_{1}$ & 0.01 & 1.0997 & 1.1644 & 1.1751 \\
$\mathrm{DC} \mathrm{MG}_{1}$ & 0.30 & 1.1021 & 1.1103 & 1.2277 \\
$\mathrm{DC} \mathrm{MG}_{1}$ & 10.00 & 1.1023 & 1.1088 & 1.1161 \\
$\mathrm{DC} \mathrm{MG}_{2}$ & 0.50 & 1.1022 & 1.1096 & 1.1169 \\
$\mathrm{DC} \mathrm{MG}_{2}$ & 14.00 & 1.1023 & 1.1088 & 1.1161 \\
$\mathrm{DC} \mathrm{MG}$ & 1.00 & 1.1022 & 1.1091 & 1.1165 \\
$\mathrm{DC} \mathrm{MG}_{3}$ & 15.00 & 1.1024 & 1.1089 & 1.1162 \\
\hline
\end{tabular}

Then, the values of $\lambda$ are calculated for each line, and thus, the values of $K_{n}$ are determined for each DC Microgrids. Table 3 shows the values of $K_{n}$ for each scenario. Consequently, the faulty DC Microgrid is identified perfectly and the connected lines will be tripped by the SSCBs.

Table 3 The values of $K_{n}$ during different scenarios

\begin{tabular}{lllll}
\hline $\begin{array}{l}\text { Faulty DC } \\
\text { MG }\end{array}$ & $\begin{array}{l}\text { Fault } \\
\text { resistance }(\Omega)\end{array}$ & $K_{n}$ & $\begin{array}{l}\text { Isolated } \\
\text { DC MG }\end{array}$ & $\begin{array}{l}\text { Operation } \\
\text { time }(\mathrm{ms})\end{array}$ \\
\cline { 2 - 4 } $\mathrm{DC} \mathrm{MG}_{1}$ & 0.01 & -1 & $\mathrm{DC} \mathrm{MG}_{1}$ & 5.50 \\
$\mathrm{DC} \mathrm{MG}_{1}$ & 0.30 & -1 & $\mathrm{DC} \mathrm{MG}_{1}$ & 19.5 \\
$\mathrm{DC} \mathrm{MG}_{1}$ & 10.00 & -1 & $\mathrm{DC} \mathrm{MG}_{1}$ & 55.4 \\
$\mathrm{DC} \mathrm{MG}_{2}$ & 0.50 & -2 & $\mathrm{DC} \mathrm{MG}_{2}$ & 5.30 \\
$\mathrm{DC} \mathrm{MG}_{2}$ & 14.00 & -2 & $\mathrm{DC} \mathrm{MG}_{2}$ & 35.0 \\
$\mathrm{DC} \mathrm{MG}_{3}$ & 1.00 & -3 & $\mathrm{DC} \mathrm{MG}_{3}$ & 20.0 \\
$\mathrm{DC} \mathrm{MG}_{3}$ & 15.00 & -3 & $\mathrm{DC} \mathrm{MG}_{3}$ & 25.1 \\
\hline
\end{tabular}

Based on Table 3, the proposed method detects the faulty DC Microgrid for fault resistances between 0 and $15 \Omega$ in less than $60 \mathrm{~ms}$. Therefore, the fault current to the faulty DC Microgrid is tripped from other connected DC Microgrids, and the faulty DC Microgrid will transferred to islanded mode. Moreover, a communication delay of $5 \mathrm{~ms}$ [30] is added to the operation time of the proposed method to consider the communication delay. Consequently, the proposed method detects the HIFs and isolates the fault DC Microgrid within an appropriate operation time. . In addition, the variations of the $\triangle D S P I$ of all lines in term of changing the fault resistance is shown in Fig 7. Thus, by increasing the value of fault resistance the value of $\triangle D S P I$ will tend to a constant value, which is higher than the threshold. Therefore, the proposed method protects system during HIFs.

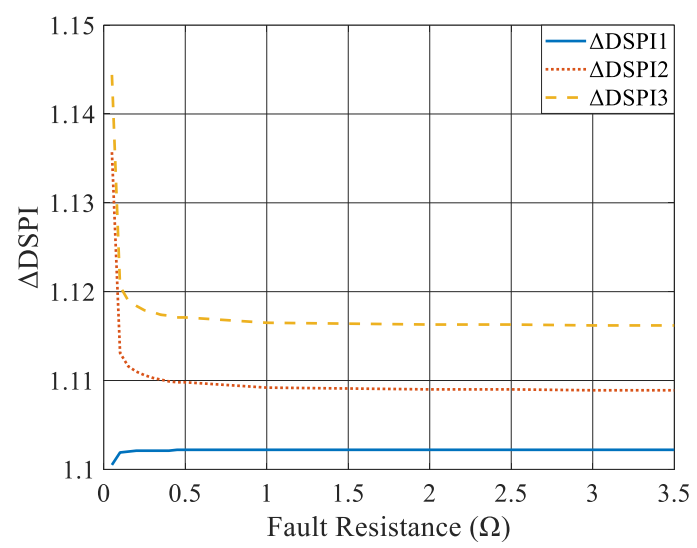

Fig. 7 The variations of $\triangle D S P I$ by changing fault resistance

\section{Conclusion}

This paper is motivated by the lack of a comprehensive and sensitive protection strategy for the interconnected DC Microgrids. The proposed and designed protection strategy is based on the DSPI and directional of current during the fault. Determining the value of fault imposed current and using DSPI enhances the sensitivity of the protection system to solve the problem of low changes during the HIFs. The proposed protection strategy consists of a relay, measurement unit, $\mathrm{SSCB}$, and a CPU, which is connected to the relays by a communication link. In this paper, the effectiveness of the proposed method is tested under different scenarios in simulation, and the results show that the proposed strategy detects HIF faults up to $15 \Omega$, and perfectly identifies and isolates the faulty DC Microgrid.

\section{References}

[1] Z. Li and M. Shahidehpour, "Small-signal modeling and stability analysis of hybrid AC/DC microgrids," IEEE Trans. Smart Grid, vol. 10, no. 2, pp. 2080-2095, Mar. 2019.

[2] Bayati, Navid, Amin Hajizadeh, and Mohsen Soltani. "Protection in DC microgrids: a comparative review." IET Smart Grid 1.3 (2018): 66-75.

[3] Parise, Giuseppe, et al. "Designing a power control strategy in a microgrid using PID/fuzzy controller based on battery energy storage." 2017 IEEE International Conference on Environment and Electrical Engineering and 2017 IEEE Industrial and Commercial Power Systems Europe (EEEIC/I\&CPS Europe). IEEE, 2017. 
[4] Bayati, Navid, Amin Hajizadeh, and Mohsen Soltani. "Accurate Modeling of DC Microgrid for Fault and Protection Studies." 2018 International Conference on Smart Energy Systems and Technologies (SEST). IEEE, 2018.

[5] Arani, Ali Asghar Khodadoost, et al. "Fault Current Limiter optimal sizing considering different Microgrid operational modes using Bat and Cuckoo Search Algorithm." Archives of Electrical Engineering 67.2 (2018): 321-332.

[6] S. D. A. Fletcher, P. J. Norman, S. J. Galloway, and G. M. Burt, "Analysis of the effectiveness of non-unit protection methods within DC microgrids," in IET Conference on Renewable Power Generation, Sept. 2011.

[7] Bayati, Navid, Amin Hajizadeh, and Mohsen Soltani. "Localized Fault Protection in the DC Microgrids with Ring Configuration." 2019 IEEE 28th International Symposium on Industrial Electronics (ISIE). IEEE, 2019.

[8] A. A. S. Emhemed, K. Fong, S. Fletcher, and G. M. Burt, "Validation of fast and selective protection scheme for an LVDC distribution network," IEEE Trans. Power Del., vol. 32, no. 3, pp. 1432-1440, Jun. 2017.

[9] Meghwani, A., S. C. Srivastava, and S. Chakrabarti. "A non-unit protection scheme for DC microgrid based on local measurements." IEEE Transactions on Power Delivery 32.1 (2016): 172-181.

[10] K. A. Saleh, A. Hooshyar, and E. F. El-Saadany, "Hybrid passive overcurrent relay for detection of faults in low-voltage DC grids," IEEE Trans. Smart Grid, vol. 8, no. 3, pp. 1129-1138, May 2017.

[11] J. D. Park, J. Candelaria, L. Ma, and K. Dunn, "DC ring-bus microgrid fault protection and identification of fault location," IEEE Trans. Power Del., vol. 28, no. 4, pp. 25742584, Oct. 2013.

[12] Bayati, Navid, Amin Hajizadeh, and Mohsen Soltani. "Fault Analysis and Protection of Low-Voltage DC Microgrid Equipped by Renewable Energy Resources." Handbook of Research on Recent Developments in Electrical and Mechanical Engineering. IGI global, 2020. 341-375.

[13] A. A. S. Emhemed and G. M. Burt, "An advanced protection scheme for enabling an LVDC last mile distribution network," IEEE Trans. Smart Grid, vol. 5, no. 5, pp. 26022609, Sept. 2014.

[14] E. Christopher, M. Sumner, D. Thomas, X. Wang, and d. W. Frans, "Fault location in a zonal DC marine power system using active impedance estimation," in IEEE Energy Conversion Congress and Exposition, Sept. 2010, pp. 30503054 .

[15] S. D. A. Fletcher, P. J. Norman, K. Fong, S. J. Galloway, and G. M. Burt, "High-speed differential protection for smart DC distribution systems," IEEE Trans. Smart Grid, vol. 5, no. 5, pp. 2610-2617, Sept. 2014.

[16] R. Mohanty and A. K. Pradhan, "Protection of smart DC microgrid with ring configuration using parameter estimation approach," IEEE Trans. Smart Grid, vol. 9, no. 6, pp. 6328-6337, Nov. 2018.

[17] S. Dhar, R. K. Patnaik, and P. K. Dash, "Fault detection and location of photovoltaic based DC microgrid using differential protection strategy," IEEE Trans. Smart Grid, vol. 9, no. 5, pp. 4303-4312, Sep. 2018.

[18] S. Dhar and P. K. Dash, "Differential current-based fault protection with adaptive threshold for multiple PV-based DC microgrid," IET Renew. Power Gen., vol. 11, no. 6, pp. 778-790, 2017.

[19] A. A. S. Emhemed, K. Fong, S. Fletcher, and G. M. Burt, "Validation of fast and selective protection scheme for an LVDC distribution network," IEEE Trans. Power Del., vol. 32, no. 3, pp. 1432-1440, Jun. 2017.

[20] B. Fani, H. Bisheh, and I. Sadeghkhani, "Protection coordination scheme for distribution networks with high penetration of photovoltaic generators," IET Gener. Transm. Distrib., vol. 12, no. 8, pp. 1802-1814, 2018.

[21] R. Mohanty and A. K. Pradhan, "Protection of smart DC microgrid with ring configuration using parameter estimation approach," IEEE Trans. Smart Grid, vol. 9, no. 6, pp. 6328-6337, Nov. 2018.

[22] R. Mohanty and A. K. Pradhan, "Protection of DC and hybrid AC-DC microgrids with ring configuration," in 7th International Conference on Power Systems, Pune, India, Dec. 2017.

[23] G. Turner, J. P. Kelley, C. L. Storm, D. A. Wetz, and W.-J. Lee, "Design and active control of a microgrid testbed," IEEE Trans. Smart Grid, vol. 6, no. 1, pp. 73-81, Jan. 2015.

[24] C. M. Colson and M. H. Nehrir, "Comprehensive real-time microgrid power management and control with distributed agents," IEEE Trans. Smart Grid, vol. 4, no. 1, pp. 617-627, Mar. 2013.

[25] Bayati, Navid, Amin Hajizadeh, and Mohsen Soltani. "Impact of Faults and Protection Methods on DC Microgrids Operation." 2018 IEEE International Conference on Environment and Electrical Engineering and 2018 IEEE Industrial and Commercial Power Systems Europe (EEEIC/I\&CPS Europe). IEEE, 2018.

[26] Kermani, Mostafa. "Transient voltage and frequency stability of an isolated microgrid based on energy storage systems." 2016 IEEE 16th International Conference on Environment and Electrical Engineering (EEEIC). IEEE, 2016.

[27] Martín-Legendre, Juan-Ignacio. "The challenge of measuring poverty and inequality: a comparative analysis of the main indicators." European Journal of Government and Economics 7.1 (2018): 24-43.

[28] M. A. Zamani, A. Yazdani, and T. S. Sidhu, “A communication-assisted protection strategy for inverter-based medium-voltage microgrids," IEEE Trans. Smart Grid, vol. 3, no. 4, pp. 2088-2099, Dec. 2012.

[29] G. Benmouy and J. Roberts, "Superimposed quantities: Their true nature and application in relays, schweitzer engineering laboratories," Inc. Pullman, WA USA, SEL USA, 199

[30] Huang C, Li F, Ding T, Jiang Y, Guo J, Liu Y. A bounded model of the communication delay for system integrity protection schemes. IEEE Transactions on Power Delivery. 2016 Feb 19;31(4):1921-33 